\title{
Spinal Cord Editor's Page October 2007
}

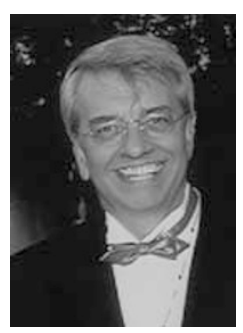

\section{Dear Spinal Cord reader,}

This issue contains a review article that investigates the objective evidence of benefits derived from functional electrical stimulation (FES)-assisted gait for people with spinal cord injury (SCI). During the ISCOS annual meeting in Iceland this year, the Guttmann lecture was on the topic of FES and was given masterly by Kristjan T Ragnarsson. As he told us: 'Repair of the injured spinal cord by regeneration therapy remains an elusive goal. In contrast, progress in medical care and rehabilitation has resulted in improved health and function of persons with SCI. In the absence of a cure, raising the level of achievable function in mobility and self-care will first and foremost depend on creative use of the rapidly advancing technology that has been so widely applied in our society. Building on achievements in microelectronics, microprocessing and neuroscience, rehabilitation medicine scientists have succeeded in developing FES systems that enable certain individuals with SCI to use their paralyzed hands, arms, trunk, legs and diaphragm for functional purposes and gain a degree of control over bladder and bowel evacuation.'

Both FES and gait have been proposed to promote not only augmented health and fitness, but also specific ambulatory outcomes for individuals with neurological disabilities. However, due to small sample sizes and the lack of functionality of the intervention, it has not been widely used in clinical practice. The review presented in this issue assessed whether there is sufficient evidence to encourage a more widespread deployment of FES gait within the rehabilitation community. A huge literature survey was done. The conclusions are interesting and evaluate whether literature on FES gait permits one to conclude that it can enhance gait, muscle strength and cardiorespiratory fitness for people with SCI. It also evaluates whether these benefits are dependent on the nature of the injury.

In developing countries, a lot of good work is done with sometimes limited means, showing how truly universal SCI management has become. An observational study by Rathore and coworkers gives us information on SCI management after the earthquake in Pakistan in 2005. It proposes how a very high number of new cases can be dealt with after such a disaster. Gupta and co-workers give the results of a postal survey on morbidity in SCI patients in India.

Tang and co-workers present a very interesting manuscript on metastatic spinal cord compression and studied whether these patients make significant functional gains through rehabilitation.

Krassioukow and co-workers present histopathologic observations in SCI in humans and sprouting of CGRP fibers. The link to developing symptoms is interesting.

There are three special case reports in this issue: on blunt cervical spine trauma and delayed cortical blindness, on acute transverse myelitis induced by campylobacter jejuni and on ultraearly MRI showing no abnormality in a fall victim with tetraparesis.

Enjoy reading and if you are working on a manuscript yourself do not hesitate to submit it for consideration for publication in Spinal Cord - 'The International Voice of the Spinal Cord'.

Spinal Cord (2007) 45, 645; doi:10.1038/sj.sc.3102126

JJ Wyndaele

Editor-in-Chief

E-mail: spinalcord@uza.be 